\title{
The Sydney University Stellar Interferometer: A Major Upgrade to Spectral Coverage and Performance
}

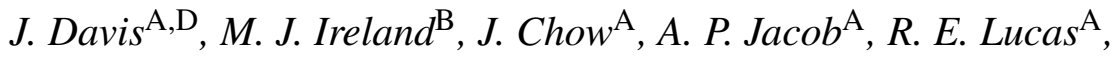 \\ J. R. North ${ }^{\mathrm{A}}, J$. W. O'Byrne ${ }^{\mathrm{A}}, S . M$. Owens $^{\mathrm{A}}, J$. G. Robertson $^{\mathrm{A}}$, \\ E. B. Seneta ${ }^{\mathrm{C}}$,W. J. Tango ${ }^{\mathrm{A}}$, and P. G. Tuthill ${ }^{\mathrm{A}}$ \\ A School of Physics, University of Sydney, Sydney NSW 2006, Australia \\ ${ }^{B}$ Planetary Science, MS 150-21, Caltech, 1200 E. California Blvd, Pasadena, CA 91125, USA \\ C Astrophysics Group, Cavendish Laboratory, Cambridge University, Cambridge, CB3 OHE, UK \\ ${ }^{\mathrm{D}}$ Corresponding author. Email: j.davis@ $@$ physics.usyd.edu.au
}

Received 2007 May 17, accepted 2007 August 31

\begin{abstract}
A new beam-combination and detection system has been installed in the Sydney University Stellar Interferometer working at the red end of the visual spectrum $(\lambda \lambda 500-950 \mathrm{~nm})$ to complement the existing blue-sensitive system $(\lambda \lambda 430-520 \mathrm{~nm})$ and to provide an increase in sensitivity. Dichroic beam-splitters have been introduced to allow simultaneous observations with both spectral systems, albeit with some restriction on the spectral range of the longer wavelength system $(\lambda \lambda 550-760 \mathrm{~nm})$. The blue system has been upgraded to allow remote selection of wavelength and spectral bandpass, and to enable simultaneous operation with the red system with the latter providing fringe-envelope tracking. The new system and upgrades are described and examples of commissioning tests presented. As an illustration of the improvement in performance the measurement of the angular diameter of the southern $\mathrm{F}$ supergiant $\delta \mathrm{CMa}$ is described and compared with previous determinations.
\end{abstract}

Keywords: instrumentation: interferometers — techniques: interferometric — stars: individual ( $\delta \mathrm{CMa})$

\section{Introduction}

The Sydney University Stellar Interferometer (SUSI) (Davis et al. 1999a) is a long baseline optical interferometer located at the Paul Wild Observatory of the Commonwealth Scientific and Industrial Research Organisation (CSIRO). The observatory is approximately $400 \mathrm{~km} \mathrm{NNW}$ of Sydney at latitude $-30^{\circ} 19^{\prime}$ and longitude $149^{\circ} 34^{\prime}$ East. SUSI was designed to have two beam-combining systems, one operating in the blue part of the spectrum and the second optimised for the red. In its initial configuration only the blue sensitive system was implemented. This was designed to operate in the spectral range $\lambda \lambda 430-520 \mathrm{~nm}$ and was restricted to using narrow spectral bandwidths $(\leq 4 \mathrm{~nm})$ with a limiting magnitude of $B \sim+2.5$.

The provision of two separate optical tables meant that a new beam-combination and detection system could be developed and installed without interrupting the observing programme of bright early-type binary stars being carried out with the original blue system. The primary motivation for the development of the new system was to significantly improve the sensitivity by using a different detection technique, more sensitive detectors, and to take advantage of the reduced effects of atmospheric turbulence at longer wavelengths. Therefore the new beam-combination system has been designed for the red end of the visual spectrum $(\lambda \lambda 500-950 \mathrm{~nm})$. The system, for which a brief outline has been given by Tuthill et al. (2004), has been commissioned and is now being employed in a multifaceted observing programme. We will refer to it as the 'red' system to distinguish it from the 'blue' system. The increased sensitivity is discussed in Section 6.3. It has significantly improved the calibration of the visibility measurements, and hence the determination of angular diameters, and it has enabled the measurement of later spectral types than is possible with the blue system.

On completion of the commissioning of the red system attention was given to upgrading the performance of the blue system and this has been described briefly by Davis et al. (2006). Dichroic beam-splitters have been introduced to split the incoming starlight between the red and blue systems. The blue beam-combination system has been reorganised with additional facilities to allow it to be used in parallel with the red system with the latter providing delay tracking for both systems. The changes are described in more detail in Section 6.2.

\section{The Red Beam-Combination and Detection System}

The overall layout of the SUSI optical system is shown schematically in Figure 1. The layout is essentially the same as that shown in Figure 2 of Davis et al. (1999a) with some small but significant changes. An additional 


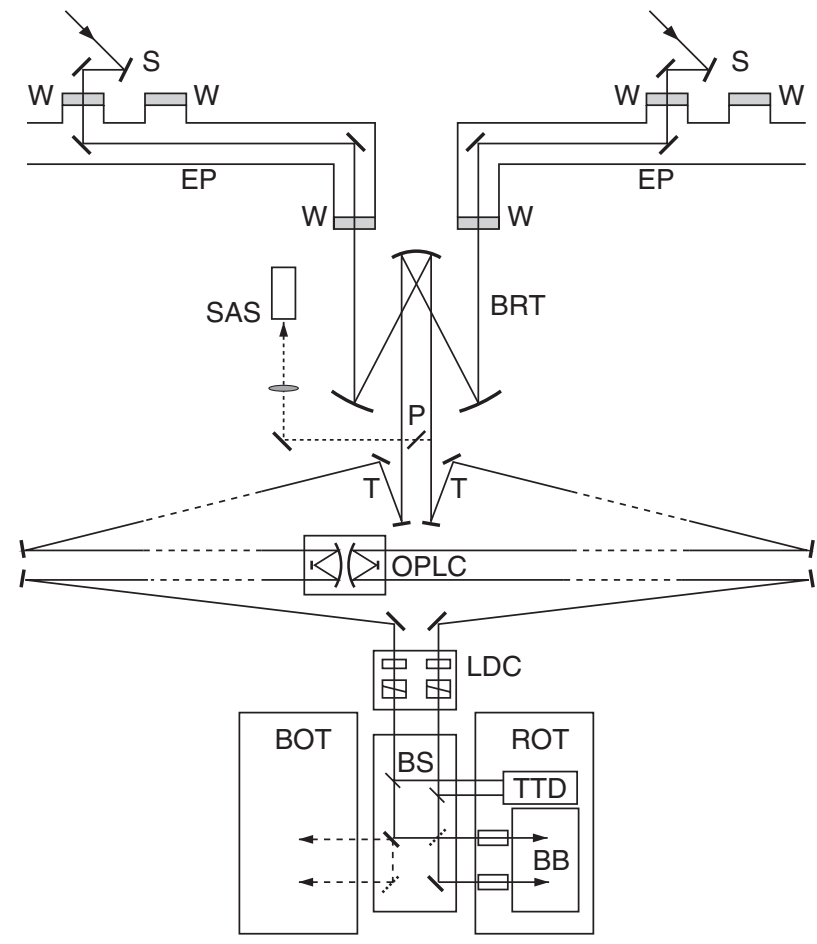

Figure 1 The overall layout of the optics. Key: S: $200 \mathrm{~mm}$ siderostat mirror (only two stations shown); W: 180-mm diameter window; EP: evacuated pipe; BRT: beam-reducing telescope; P: pellicle; SAS: star acquisition system; T: wavefront tip-tilt correcting mirror; OPLC: optical-path-length compensation carriage; LDC: longitudinal dispersion corrector; BS: beam-splitters; BOT: 'blue' optical table; ROT: 'red' optical table; BB: optical bread-board for 'red' beam-combination optics; TTD: wavefront tip-tilt detector (see Figure 3 for more detail). Further details are given in the text.

reflection has been introduced in the south beam prior to the beam-reducing telescope (not shown in the figure) to make the polarisation properties of the two arms symmetrical and some changes have been made to the layout of the optical path length compensator (OPLC) to reduce the number of reflections. The two beams of starlight from the north and south arms of the baseline array, after passing through the OPLC and the longitudinal dispersion corrector (LDC), are relayed to the platform between the two optical tables as shown in Figure 1. We note that the LDC, which was described by Davis et al. (1999a), is operated in 'single-glass' mode with only the BK7 glass as the compensating medium when used with the red system. This is possible because the dispersion is significantly less in the red than in the blue region of the spectrum. The most significant change from figure 2 of Davis et al. (1999a) is the inclusion of the second optical table which illustrates the relative locations of the red and blue beam-combination systems. Initially, starlight was directed to either the blue or red table by manually changing the mirrors on the central pier as shown in Figure 1.

The layout of the optics for the red system basically follows that for the blue system Davis et al. (1999a) and is shown schematically in Figure 2. There are, however, a few key differences. These include a new wavefront tip-tilt

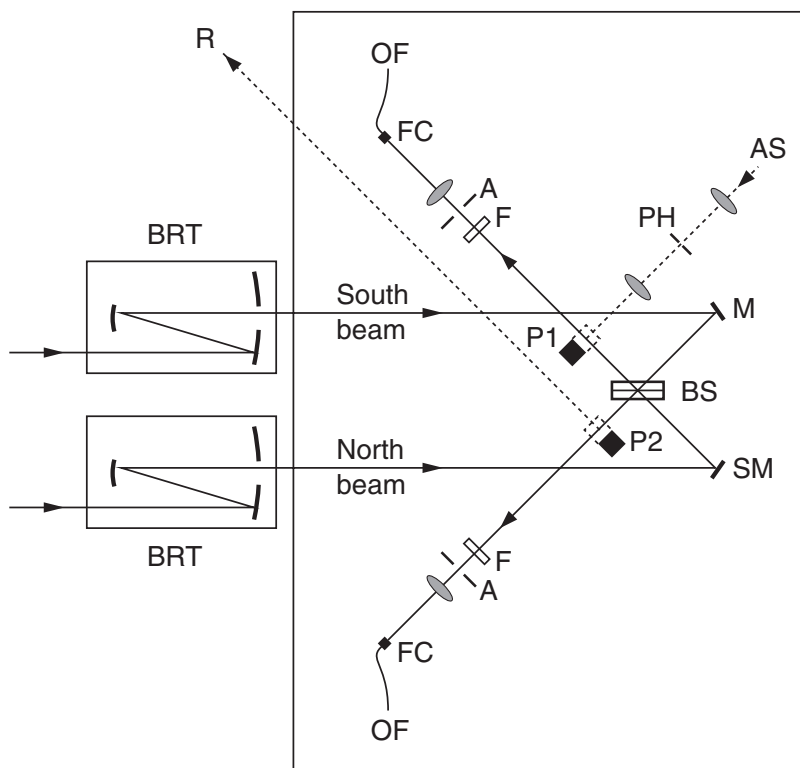

Figure 2 A schematic of the layout of the red system optics. The large rectangle is the outline of the optical breadboard shown in Figure 1. Key: BRT: beam-reducing telescope; M: fixed mirror; SM: scanning mirror; BS: beamsplitter; P1 and P2: slide-mounted pentaprisms; F: filter; A: aperture; FC: optical fibre clutch; OF: optical fibre; PH: pinhole; AS: artificial 'star' light source; R: reference beam. Further details are given in the text.

detection system based on a CCD camera, additional beam reducing telescopes at the input to the beam-combination system to allow for a more compact system and to provide a location for field stops or spatial filters, wide-band spectral filters combined with a fringe-envelope scanning mirror to increase the sensitivity, and avalanche photodiode (APD) detectors in place of photomultipliers to give high detected quantum efficiency in the red region of the spectrum. These key changes are discussed in the following sections. The introduction of dichroic beam-splitters and the rearrangement of the blue system is discussed in Section 6.2.

\subsection{The Optical System}

The two beams of starlight from the north and south arms, on reaching the central pier, are first divided by partially polarising beam-splitters (mean reflectance at $45^{\circ}$ for $\lambda \lambda$ $440-900 \mathrm{~nm}$ equals 0.64 for the s-polarisation and 0.36 for the p-polarisation giving a mean reflectance of 0.5 ). The reflected beams are focussed by a Celestron 8 telescope onto the chip of a CCD camera mounted on the red optical table. Apertures in front of the telescope define the beam size. The images are used to detect atmospheric turbulence induced wavefront tilts and to provide feedback to the tip-tilt mirrors (see Section 2.3). The transmitted beams pass through apertures to define the size of the signal beams. Aperture diameters of $28 \mathrm{~mm}$ are generally employed (equivalent to $84 \mathrm{~mm}$ on the sky). The beams are then reflected by mirrors onto the red table where the beam diameters are reduced by individual beam-reducing 
telescopes. Each of the beam-reducing telescopes consists of two concave paraboloidal mirrors arranged in a confocal configuration to produce a collimated output. The primary mirrors have focal lengths of $405 \mathrm{~mm}$ and the secondaries focal lengths of $98.5 \mathrm{~mm}$ giving a reduction in the beam diameter by a factor of 4.1 . The primary mirror of each telescope has a hole trepanned in its centre. The input beam is incident on the primary mirror to one side of the central hole and the output beam reflected from the secondary mirror passes through the central hole in the primary. Pinholes can be placed at the common foci of the paraboloidal mirrors to act as field stops for isolating individual stars of visual binaries or for spatial filtering. The beams exiting the beam-reducing telescopes enter a beam-combination system with the layout shown in schematic form in Figure 2. Mirrors reflect the incoming beams into a partially reflecting dielectric beam-splitter at $45^{\circ}$. The reflectivity of the beam-splitter is within $10 \%$ of 0.50 over the spectral range $500-900 \mathrm{~nm}$ at the design angle of $45^{\circ}$ for the polarisation with the E-field horizontal. A major difference from the blue system is that the mirror reflecting the north beam into the beam-splitter is mounted on a piezo-actuated linear translator that allows the mirror to be scanned through the fringe envelope. The combined beams exiting on the two sides of the beamsplitter pass through matched broad-band interference filters before being focussed as $f / 12$ beams on $100-\mu \mathrm{m}$ diameter multimode optical fibre feeds to APD detectors located on the roof of the instrument enclosure. There is provision for the insertion of wire grating polarisers prior to the focussing lenses. The matched interference filters are mounted in filter wheels so that it is possible to switch rapidly between filters with different central wavelengths and spectral bandwidths. Several pairs of matched broadband filters (with spectral bandwidths of $~ 5 \%$ and 10\%) are available but most observations to date, including those presented here, have been made using filters centred on $700 \mathrm{~nm}$ with a spectral bandwidth of $80 \mathrm{~nm}$.

\subsubsection{Alignment Aids}

The critical alignment of a long-baseline interferometer and the alignment aids and techniques employed in SUSI for the blue beam-combining optics have been discussed by Davis et al. (1999a). Similar additional optics and an 'artificial star', identified in Figure 2 by AS, have been included for the red table. The artificial star is a diffraction-limited pinhole that can be illuminated with a 633-nm HeNe laser for alignment purposes or by a Maglite torch bulb (manufactured by Mag Instruments Inc.) that acts as a white light source for establishing matched path positions through the white light fringe. Pentaprisms are used instead of the periscope rhombs used on the blue table Davis et al. (1999a) and these are mounted on precision slides driven by linear stepping motors with Hall effect position sensors. They are used to insert the laser or white light beams into the beam-splitter along the optical axis of the interferometer or to relay a reference beam of incoming starlight to the CCD based tip-tilt detection system. An additional pentaprism, not shown in Figure 2, is used to reflect light to a CCD video camera to assist with alignment.

Two improvements have been made that do not affect the sensitivity of the instrument but which have facilitated alignment and improved the reliability and efficiency of operation. They are the installation of a new local area network and the introduction of a large number of New Focus picomotor drives in place of micrometers for remote mirror and beam-splitter control. The picomotors are operated through the network from a handterminal that can be patched to any location within the instrument and this enables alignment to be performed efficiently by one person. The crucial beam-splitters that align the tip-tilt images relative to the reference images to ensure accurate overlap of the science beams are equipped with picomotors and, as well as being adjustable via the hand terminal, they are computer-controlled to permit automatic alignment with starlight during observing sessions.

\subsection{The Star-Acquisition System}

The SUSI star-acquisition system has been described by Davis et al. (1999a) and in detail by Seneta (1991). A pellicle beamsplitter can be moved into either the north or south beam to reflect $\sim 8 \%$ of the incident light to an intensified CCD camera. Originally the camera fed a video signal to custom hardware that provided the logic for determining the pointing error in real time. The hardware was interfaced with a single-board computer and an MS-DOS based PC to provide feedback signals to correct siderostat pointing during star acquisition. To overcome problems with the now obsolete video digitiser chip the system has been upgraded and the video signal now goes directly to a 'framegrabber' card installed in a PC running Microsoft Windows. The logic is now integrated into new software that displays the video signal and links the acquisition system directly with the new tip-tilt servo.

\subsection{Tip-Tilt Correction}

The main components of the new tip-tilt system are the CCD detector shown in Figure 3 and the tip-tilt mirrors labelled $\mathrm{T}$ in Figure 1. The tip-tilt mirrors are activated by two-axis piezo-electric actuators. Two Astromed/Astrocam cameras have been used as the detector: the original one with an EEV 30-A CCD chip and a newer one with a back-illuminated SITe 502A chip. The overall control of the system is managed by a PC running real-time Linux.

The CCD accepts broad-band light which, in practice, means that wavelengths between 400 and $950 \mathrm{~nm}$ are used for tip-tilt detection. The broad optical bandwidth is employed so that adequate photon count rates can be obtained when observing relatively faint stars. There are no atmospheric refraction correctors so the width and 


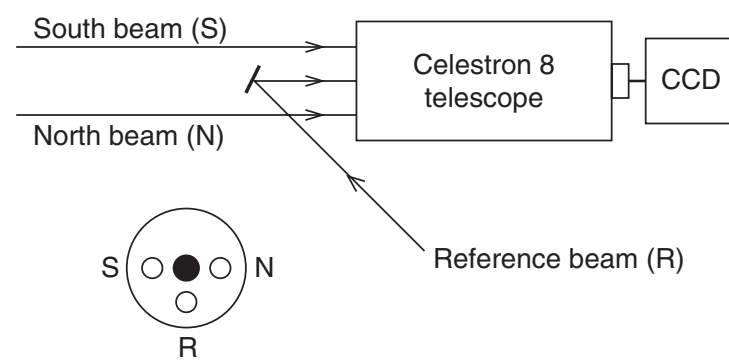

Telescope aperture

Figure 3 Schematic layout of the wavefront tip-tilt detection system. The relative positions of the input beams on the aperture of the telescope are shown in the lower diagram. Further details are given in the text.

shape of the point-spread functions used for tip-tilt tracking are a function of seeing, stellar spectral type, and the zenith-distance of the source.

The configuration of the three image readout regions in the corner of the CCD nearest to its readout region is shown in Figure 4. In this standard observing mode the pixels are square with each side equivalent to 0.81 arcsec on the sky. The 5 pixel by 5 pixel readout regions for the north and south beams enable the stellar point spread functions to be sampled. This in turn enables centroid estimation that is less dependent on the shape of the point-spread function than for a quad-cell centroid estimator. All images and tip-tilt mirror positions are saved during observing and the data sets are used to estimate residual seeing effects in post-observational processing. A $20 \mathrm{~ms}$ sample time is generally used for observing in this configuration, although a $10 \mathrm{~ms}$ sample time is available for the (rarely used) quad-cell 2-pixel by 2-pixel binning mode. These relatively long sample times seriously limit the tip-tilt performance in fast seeing conditions, but are necessary because of long CCD readout times and the requirement for similar tip-tilt performance on both bright and fainter stars. A new camera incorporating an electron-multiplying $\mathrm{CCD}$ (EMCCD) is being commissioned to give improved signal-to-noise and faster read-out.

Fine alignment of the tip-tilt system is achieved using the reference beam labelled $\mathrm{R}$ in Figures 2 and 3. This beam travels through a 4:1 beam expander before being imaged by the Celestron 8 telescope onto the 4 pixel by 4 pixel reference readout region shown in Figure 4. While the tip-tilt servo is locked, the north and south beams are viewed one at a time in the reference region by closing and opening appropriate shutters on the red table. Picomotors adjust the orientation of the north and south tip-tilt beamsplitters so that the images formed by the north and south beams are individually centred in the reference region. This ensures that the north and south beams are overlapped with their wavefronts plane parallel at the beam-combiner (BS in Figure 2). The tip-tilt servo, picomotor and red table slide and shutter controllers are all connected to the local area network, enabling alignment of the tip-tilt system to be performed automatically.

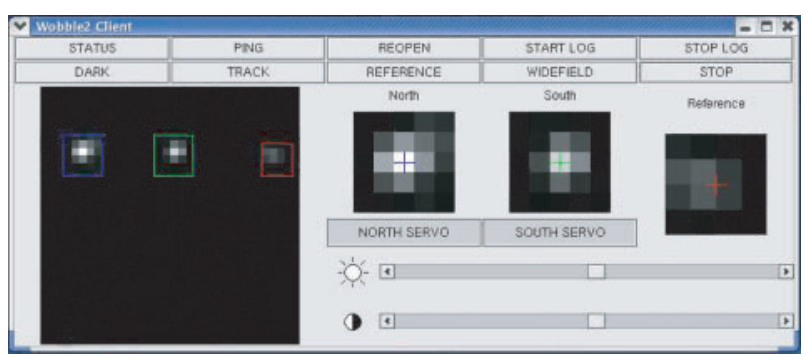

Figure 4 The graphical user interface for the new tip-tilt system, showing the images for the servos locked on a 633-nm laser signal autocollimated from the siderostats.

\subsection{The Optical-Path-Length Compensation (OPLC) System}

The OPLC system in SUSI has been described in detail by Davis et al. (1999a). In brief, a carriage carrying back-to-back cat's eye retro-reflectors moves differentially between starlight from the north and south beams over a 70-m long track. The position and velocity of the carriage are monitored and controlled by the difference signal between laser metrology systems mounted at each end of the track. A number of improvements have been made to the OPLC system. These include:

- The system has been ported and streamlined from the original, now obsolete, Solaris workstation and VME crate to a PC running Linux.

- The control and user interface software has been upgraded and communication has been established with the fringe-detection system to allow feedback of the current fringe-envelope centre position and the servoing of the carriage position (Section 2.5).

- The original long and short low-voltage piezo-electric actuators (PEAs) on which the small flat mirrors at the foci of the cat's eye retro-reflectors were mounted have been replaced by two nominally identical PEAs. The new PEAs act in a push-pull arrangement, each compensating half the tracking-error signal, with an increased bandwidth of $500 \mathrm{~Hz}$. Each of the PEAs has a near identical, experimentally determined range of $78 \mu \mathrm{m}$ in optical path with a step resolution of $19 \mathrm{~nm}$ in optical path.

- The fact that the two PEAs are nominally identical, and have been shown to have similar expansion and hysteresis characteristics, allows operation with a single metrology laser operating from one end of the OPLC. This facility has been commissioned to provide a back-up mode in the event of a metrology laser failure.

- The stepper motor that drives the carriage along the rails has been replaced with a new stepper motor. The new motor shows lower frequency and significantly reduced RMS deviations from linearity and it follows that these are more efficiently corrected by the piezo-mounted secondary mirrors of the cat's eyes.

- The high-friction, high-rigidity tyre on the drive wheel that is directly coupled to the stepper motor has been replaced. It was found to have worn unevenly resulting 


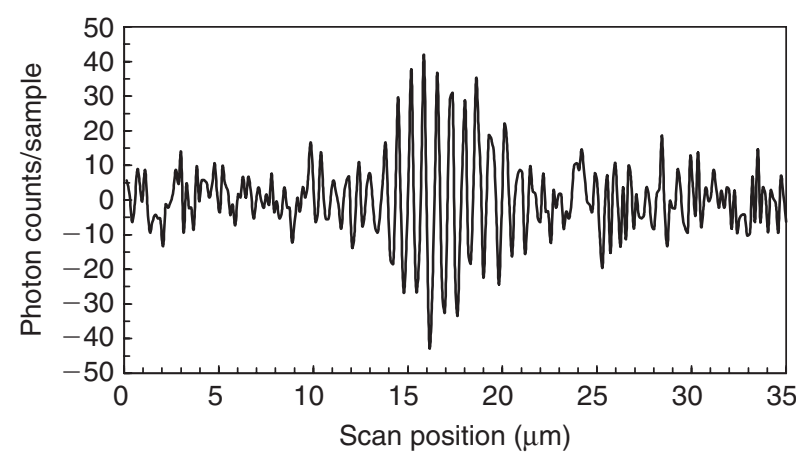

Figure 5 An example of a single short raw fringe scan for $\delta \mathrm{CMa}$ at a baseline of $5 \mathrm{~m}$ on 21 April, 2004. The photon counts/sample time are relative to the mean count for the scan and the scan consists of 256 samples each $0.2-\mathrm{ms}$ long. The mean count per sample for the scan was 43 .

in large systematic optical path tracking errors. Steel wheels of the same outside diameter as the tyre have been installed and improved tracking has been achieved.

- Non-linearities in the motor drive and in the hysteresis of the PEAs have been modelled and used to optimise the control software.

\subsection{Fringe Detection and Data Acquisition}

The fringe-detection system consists of two Perkin Elmer Photon Counting Module APD detectors (fed by the optical fibres shown in Figure 2) and a mirror (labelled SM in Figure 2) mounted on a piezo-electric actuator that scans through an optical path up to $140-\mu \mathrm{m}$ long. The actuator is driven to and fro by a triangular waveform generated from lookup tables to eliminate non-linearities in the motion. The fringe signal is the difference in count rates between the two APDs. This is similar to some features of the system developed for COAST (Baldwin et al. 1994). An example of a fringe scan using a short scan of length $35 \mu \mathrm{m}$ is shown in Figure 5. The control software for the fringe-detection and data-acquisition system runs on a real-time Linux PC. As well as the basic tasks of controlling the scanning mirror and saving the raw data streams, the control computer calculates the fringe power spectrum (shown in Figure 6), estimates the squared fringe visibility, the signal-to-noise ratio, and the fringe position.

In the low signal-to-noise regime, finding the fringe position is aided by forming the envelope of fringe power. This fringe envelope is the squared modulus of the analytic signal as defined by Bracewell (1965). It is calculated by windowing the fringe peak in the positive half of the fringe Fourier transform, forming the inverse transform and taking its squared modulus. The upper and lower bounds of the rectangular window function are shown by the dotted lines in Figure 6. A fading memory average of the fringe power spectrum and fringe-envelope function are displayed to the operator in a graphical user interface, and the first moment of the bias-subtracted fringe envelope is used for tracking the fringes. Details of the fringe-tracking algorithm are discussed by Ireland (2005).

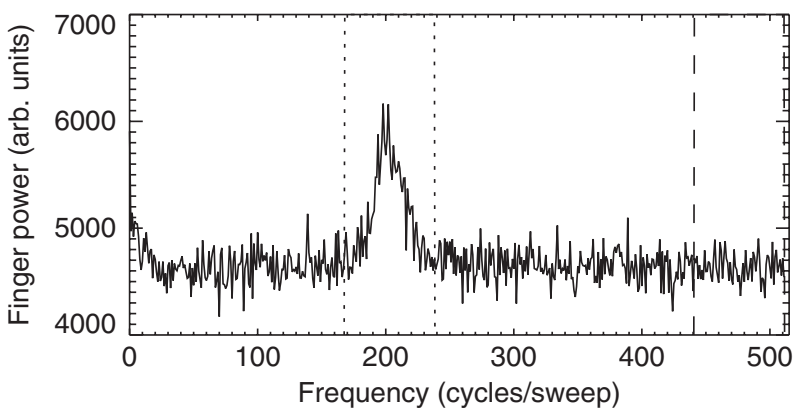

Figure 6 An integrated power spectrum of $\tau$ Vir (HR 5264) $(R$ magnitude $=4.2$ ) for 1000 fringe scans observed at a baseline of $80 \mathrm{~m}$ in $1.8 \mathrm{arcsec}$ seeing. The dotted lines show the edges of the rectangular window function (see text), and the dashed line shows the equivalent window for estimating bias. Note that the y-ordinate reaches zero well below the $\mathrm{x}$-axis, demonstrating that the fringe signal centered around 200 cycles/sweep is dominated by bias.

\section{Observing with the Red System}

The observing procedure has been automated with a software scheduler that controls the sequence of operations that constitute a complete observation. This not only eases the load on an observer but also ensures that no steps in the observing procedure are missed. Each stellar observation includes a set of fringe scans and photometric and background scans. A complete observation of a star including acquisition, fringe scans and photometric scans is completed in an average of $6 \mathrm{~min}$. Each observation of a programme star is generally interleaved between observations of 'calibrator' stars so that a sequence of 'calibrator-programme star-calibrator' is completed in $\sim 18 \mathrm{~min}$. Calibration is discussed further in Section 3.2.

\subsection{The Scheduler}

The scheduler software accepts the HR number of the star to be observed and causes an astrometric file with position and tracking velocities for the siderostats and OPLC to be created. It then initiates a sequence of operations. The siderostats and OPLC carriage are driven to their predicted positions and set moving at their tracking velocities. The north and south images from the siderostats are located successively by the star-acquisition system which provides correction signals to the siderostats to bring the images within the field of view of the tip-tilt system. At this point the tip-tilt system takes over and closes the feedback servo to the siderostats. With the tip-tilt servo on, the OPLC is scanned through a preset range to find and acquire fringes. A preset number of fringe scans are then recorded and, on completion of the scans, shutters are moved in and out of the beams to obtain photometric measurements for each beam. The siderostats are stopped and the background is measured with the star out of the field of view. The scheduler then accepts the next HR number from a command line input or from a scheduler file. The observer is alerted to any errors or problems in the sequence of operations and is able to interrupt the scheduler at any time and 
subsequently resume where it stopped. Prior to the commencement of an observing session, and approximately every hour during the session, the scheduler is instructed to initiate a fine alignment of the tip-tilt system.

\subsection{Calibration}

As described in Sections 2.5 and 4, SUSI measures the square of the interference fringe visibility $\left(V^{2}\right)$. In practice this will be less than the true $V^{2}$ due to instrumental and residual seeing losses. The magnitude of these losses is established by interleaving the observations of a programme star between observations of 'calibrators'. A calibrator is ideally a star whose true $V^{2}$ can be accurately predicted-either because it has a small angular size compared to the programme star or because its angular diameter is known accurately.

There are a number of desirable characteristics for a calibrator and these include being single, non-variable, and close to the programme star in the sky. Ideally a calibrator would be of similar magnitude and spectral type as the programme star but this implies that the stars are nearly identical and it follows that this does not make for a suitable calibrator. In practice, compromises have to be made and these depend heavily on the limiting magnitude of the instrument. The problem experienced in calibrating observations for the blue system was the fact that the relatively bright limiting magnitude made it very difficult to find suitable calibrators close to a programme star. The increase in sensitivity of the red system has eased this problem.

The calibration procedure essentially involves defining a transfer function $T$ for a calibrator by

$$
T=\frac{V_{\mathrm{obs}}^{2}}{V_{\exp }^{2}}
$$

where $V_{\mathrm{obs}}^{2}$ is the observed $V^{2}$ and $V_{\mathrm{exp}}^{2}$ is the expected $V^{2}$ for the calibrator. If $V_{\text {obs* }}^{2}$ is the observed $V^{2}$ for the programme star, its true visibility squared $V_{\text {true* }}^{2}$ is given by

$$
V_{\text {true* }}^{2}=\frac{V_{\text {obs* }}^{2}}{\bar{T}}
$$

where $\bar{T}$ is the mean of the transfer functions of calibrators observed either side of the programme star.

\subsection{The Effective Wavelength}

The effective wavelength of observations made with the red system will be a function of the spectral type of the star being observed and also the filters employed because of their wide spectral bandwidths. The effective wavelength $\lambda_{\text {eff }}$ is given by

$$
\frac{1}{\lambda_{\text {eff }}}=\sigma_{\text {eff }}=\frac{\int_{0}^{\infty} I^{2}(\sigma) \sigma \mathrm{d} \sigma}{\int_{0}^{\infty} I^{2}(\sigma) \mathrm{d} \sigma}
$$

with

$$
I^{2}(\sigma)=T(\sigma) N^{2}(\sigma) S^{2}(\sigma)
$$

where $\sigma$ is the wavenumber equal to $1 / \lambda, T(\sigma)$ is the transfer function (see equation (1)), $N(\sigma)$ is the photon flux from the star per unit wavenumber interval, and $S(\sigma)$ is the spectral response of the instrument.

The effective wavelength as a function of intrinsic $(B-V)_{0}$ for main sequence stars has been evaluated for the $700 \mathrm{~nm}$ filters via computation of the spectral response of the system and also by measurements. The spectral response $S(\sigma)$ has been computed taking into account the spectral transmission of the optical system, including window transmission, mirror reflectance, beam-splitter transmission/reflectance, transmission of the matched interference filters, the quantum efficiencies of the APDs as a function of wavenumber, and the transmission of the atmosphere. The transfer function includes the loss in $V^{2}$ due to residual seeing effects and an approximate allowance for this has been included in the computations. The losses in $V^{2}$ for no correction and for $100 \%$ correction of wavefront tip-tilt have been published by Tango \& Twiss (1980). The losses for 1.5 arcsec seeing and $~ 50 \%$ correction have been derived as a function of wavenumber from the Tango \& Twiss results as representative of average conditions for SUSI observations and used in the calculation of effective wavelength. It is noted that the extremes of 1 arcsec seeing with full tip-tilt correction and 2 arcsec seeing with no correction only change the effective wavelength by $\pm 0.06 \%$. Effective wavelengths were computed for spectral types $\mathrm{O} 5 \mathrm{~V}, \mathrm{~B} 6 \mathrm{~V}, \mathrm{~A} 2 \mathrm{~V}, \mathrm{~A} 8 \mathrm{~V}$, F3 IV, G2 IV, G8 IV, K4 V and K5 V using photon flux distributions derived from the library of stellar spectra by Silva \& Cornell (1992).

Measurements of the effective wavelength have also been made from sets of scans recorded for selected main-sequence stars. The scan steps were calibrated by means of scans taken in autocollimation with the 633-nm HeNe laser illuminating the 'artificial star' pinhole (Section 2.1.1). In the case of the stellar measurements, the power spectrum of each sweep, determined in the dataprocessing pipeline to be described in Section 4.1, is an estimate of $I^{2}|\gamma|^{2}$ where $|\gamma|$ is the degree of coherence at the observing baseline. In the pipeline the power spectra are averaged and are used to estimate the fringe visibility. The first moment of the power spectrum is

$$
\sigma_{1}=\frac{\int_{0}^{\infty} I^{2}(\sigma)|\gamma(\sigma)|^{2} \sigma \mathrm{d} \sigma}{\int_{0}^{\infty} I^{2}(\sigma)|\gamma(\sigma)|^{2} \mathrm{~d} \sigma}
$$

For observations made where $|\gamma(\sigma)|^{2} \sim 1$, we have $\sigma_{1}=\sigma_{\text {eff. }}$ The main-sequence stars selected for the measurement of $\sigma_{\text {eff }}$, and hence $\lambda_{\text {eff }}$, were observed at a baseline of $5 \mathrm{~m}$ such that this condition was met. The stars and the effective wavelengths determined for them are listed in Table 1. 
Table 1. The measured values of effective wavelength $\left(\lambda_{\text {eff }}\right)$ for selected main-sequence stars measured at a $5 \mathrm{~m}$ baseline. $N$ is the number of measurements and SEM the standard error in the mean value of effective wavelength

\begin{tabular}{lrrrr}
\hline Star & $(B-V)_{0}$ & $N$ & $\begin{array}{c}\lambda_{\text {eff }} \\
(\mathrm{nm})\end{array}$ & $\begin{array}{l}\text { SEM } \\
(\mathrm{nm})\end{array}$ \\
\hline$\tau$ Sco & -0.30 & 18 & 695.20 & 0.17 \\
$\kappa$ Vel & -0.24 & 5 & 695.93 & 0.48 \\
$\sigma$ Leo & -0.05 & 4 & 694.97 & 0.84 \\
$\alpha$ CMa & 0.00 & 8 & 693.69 & 0.34 \\
¿ Cen & 0.04 & 10 & 695.33 & 0.43 \\
$\phi$ Leo & 0.20 & 4 & 694.83 & 1.27 \\
$\beta$ TrA & 0.30 & 10 & 695.33 & 0.22 \\
$\beta$ Vir & 0.52 & 7 & 697.26 & 0.87 \\
$\beta$ Vir & 0.52 & 6 & 695.90 & 0.87 \\
\hline
\end{tabular}

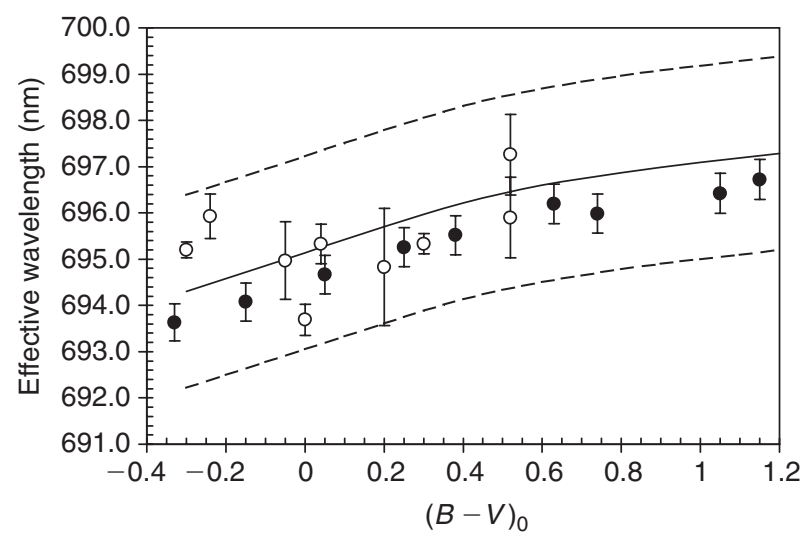

Figure 7 The computed and measured values of effective wavelength with the adopted relationship shown by the solid line. Key: - - computed values; o - measured values. The estimated uncertainty in the adopted relationship of $\pm 0.3 \%$ is represented by the two dashed lines.

The computed and measured values of $\lambda_{\text {eff }}$ are plotted against $(B-V)_{0}$ in Figure 7 . There is excellent agreement between the measured and calculated values with the mean difference equal to $\sim 0.08 \%$. The shape of a smooth curve drawn through the calculated points is insensitive to changes in the parameters that enter the calculations and only the vertical positions of the calculated values in Figure 7 are affected. The adopted scale of effective wavelength for main-sequence stars is therefore based on a smooth curve drawn through the calculated points shifted by $+0.6 \mathrm{~nm}(\sim 0.08 \%)$ to place it close to the mean difference between the measured and calculated values and this is represented by the solid line in Figure 7. A conservative uncertainty of $\pm 0.3 \%(\sim \pm 2 \mathrm{~nm})$ has been adopted and this is shown by the dashed lines in Figure 7 . The effective wavelengths corresponding to the solid line in the figure are tabulated against $(B-V)_{0}$ in Table 2 .

The effective wavelength for reddened main-sequence stars or stars of other luminosity classes, reddened or not, can be computed from the observed stellar flux distribution corrected for atmospheric extinction following the procedure described here.
Table 2. The adopted scale of effective wavelength $\lambda_{\text {eff }}$ versus $(B-V)_{0}$ for SUSI's $700 \mathrm{~nm}$ system for non-reddened main-sequence stars. Details are given in the text

\begin{tabular}{lccc}
\hline$(B-V)_{0}$ & $\begin{array}{c}\lambda_{\text {eff }} \\
(\mathrm{nm})\end{array}$ & $(B-V)_{0}$ & $\begin{array}{c}\lambda_{\text {eff }} \\
(\mathrm{nm})\end{array}$ \\
\hline-0.30 & 694.3 & 0.50 & 696.4 \\
-0.20 & 694.6 & 0.60 & 696.6 \\
-0.10 & 694.9 & 0.70 & 696.7 \\
0.00 & 695.1 & 0.80 & 696.9 \\
0.10 & 695.4 & 0.90 & 697.0 \\
0.20 & 695.7 & 1.00 & 697.1 \\
0.30 & 696.0 & 1.10 & 697.2 \\
0.40 & 696.2 & 1.20 & 697.3 \\
\hline
\end{tabular}

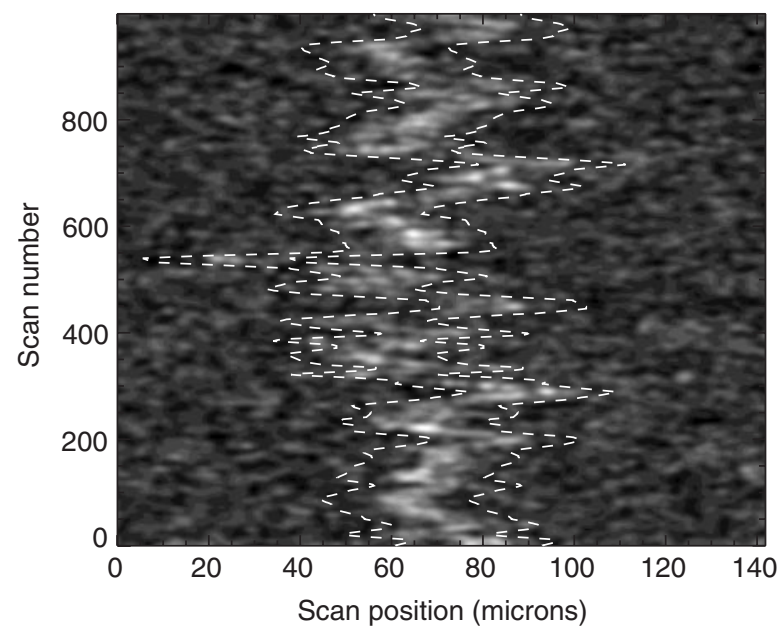

Figure 8 The smoothed fringe envelopes (see Section 4.1) for a set of 1000 scans for $80 \mathrm{~m}$ baseline data on $\tau$ Vir (HR 5264) $(R$ magnitude $=4.2)$ in 1.8 arcsec seeing. Dashed lines join the edges of the spatial-domain window. Signal-to-noise per scan is 0.55 before spatial-domain windowing and 0.8 after. The integrated power spectrum for these data is shown in Figure 6.

\section{Data Analysis}

A software pipeline has been developed to process the recorded fringe scans and this is described in the following section.

\subsection{Data Pipeline}

The data analysis pipeline first forms the power spectrum of the fringes and the fringe envelope. Once the fringe envelope has been calculated for each scan, a moving spatial-domain window function is defined as shown in Figure 8. This window is based on an estimate for the fringe position formed by looking for the peak in the set of fringe envelopes smoothed in space and time. Only the data within this spatial domain window are considered in forming the final estimate for the observed $V_{\mathrm{obs}}^{2}$. The details of the determination of $V_{\mathrm{obs}}^{2}$, which is proportional to the sum of fringe power (minus bias) normalised by the product of the mean APD fluxes, are given by Ireland (2005). 


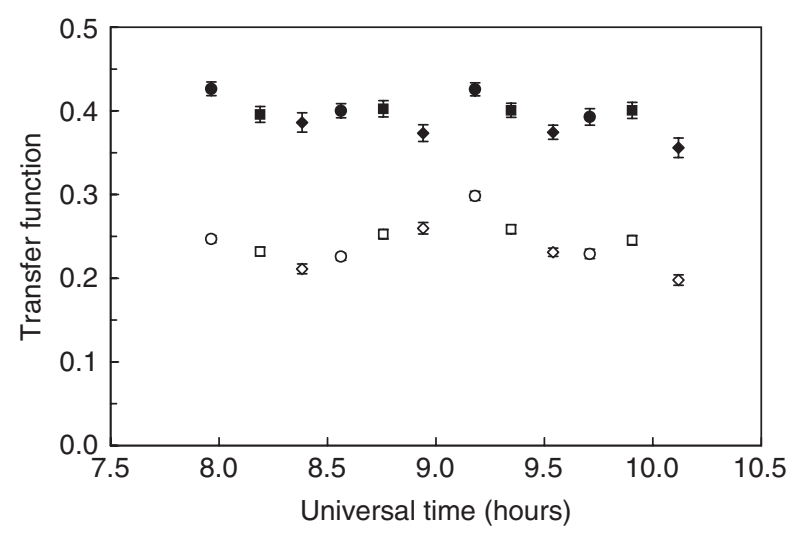

Figure 9 An example of output from the data processing pipeline for three calibrators observed at $700 \mathrm{~nm}$ with a baseline of $20 \mathrm{~m}$ on 2 July 2004. Key: round symbols are for HR 3685 ( $\beta$ Car), square symbols for HR 3699 ( $\mathrm{C}$ Car) and diamond symbols for HR 4199 ( $\theta$ Car). Open symbols represent transfer function $T$ values obtained using equation (1) and the filled symbols are for the same data but with the seeing correction of equation (6) applied.

Two important quantities are extracted from the recorded tip-tilt data. The first, $\sigma_{\mathrm{t}}^{2}$, is the variance in tiptilt system centroid, corrected for effects of photon and readout noise. The second, $\theta_{\mathrm{T}}$ is the full-width half maximum of a Gaussian fitted to the sum of the tip-tilt mirror position and the image centroid. The variance in the normalised APD count rates $\sigma_{\mathrm{I}}^{2}$ is also used as a measure of scintillation. Together, these three quantities are used to correct the value of $V_{\mathrm{obs}}^{2}$ for each data set:

$$
V_{\text {obs_corr }}^{2}=V_{\text {obs }}^{2} \exp \left(a \sigma_{\mathrm{t}}^{2}+b \theta_{\mathrm{T}}^{2}+c \sigma_{\mathrm{I}}^{2}\right)
$$

Here $V_{\text {obs_corr }}^{2}$ is the value of $V_{\text {obs }}^{2}$ corrected for residual seeing, and the parameters $a, b$ and $c$ have been estimated empirically based on fits to large data sets of calibrator observations. Further details of this seeing correction can be found in Ireland $(2005,2006)$. The procedure has produced a significant improvement in the calibration of the observational data. An example of data processed with and without the seeing correction for three calibrators observed on 2 July, 2004, a night of variable seeing, is shown in Figure 9. The overall scatter in the transfer function about the mean value has been reduced from $\pm 21 \%$ to $\pm 9 \%$ for this data set. The improvement achieved depends on the quality of the night but, taking an average over several nights, the application of the seeing correction reduced the scatter in the transfer function by a factor of $\sim 1.65$.

\section{The Blue-System Upgrade}

Following the completion of commissioning of the red system, dichroic beam-splitters were introduced on the central pier, as shown in Figure 10, to split the incoming starlight between the blue and red tables to enable them to be used simultaneously. The blue end of the spectrum, for wavelengths up to $520 \mathrm{~nm}$, is transmitted while the red end of the spectrum is restricted to $550-760 \mathrm{~nm}$ for $>95 \%$ reflectance. The dichroic beam-splitters are designed and

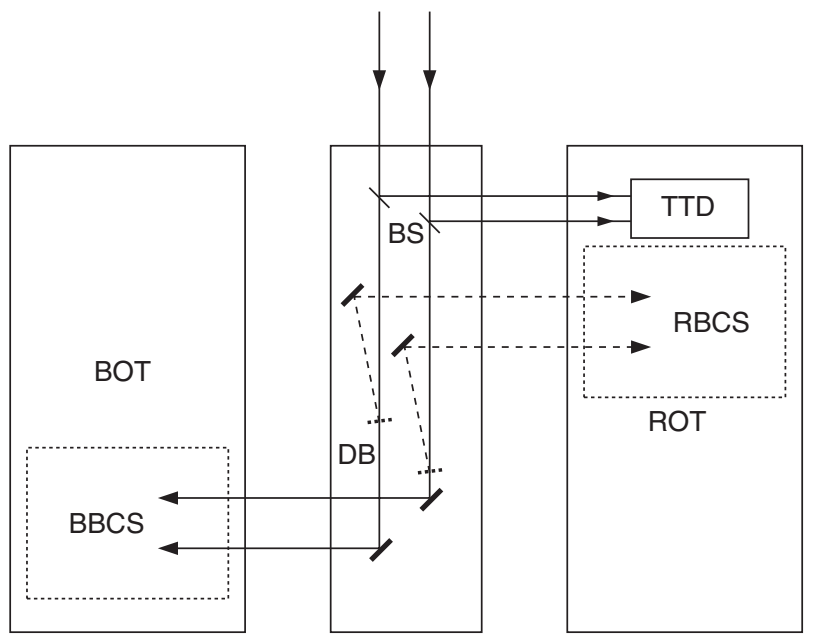

Figure 10 The layout of the beam-relay system for the red and blue beam-combining systems. Key: $\mathrm{BS}=$ Beam-Splitters; $\mathrm{TTD}=$ Tip-Tilt Detector; $\mathrm{DB}=$ Dichroic Beam-splitters; $\mathrm{BOT}=$ Blue Optical Table; $\mathrm{BBCS}=$ Blue Beam-Combining System; ROT $=$ Red Optical Table; RBCS $=$ Red Beam-Combining System.

oriented for near-normal incidence of the incoming beams to minimize differential polarisation effects.

The original blue system has been rearranged to accommodate the change in position at which the north and south beams are directed onto the table. The polarising beam-splitter cubes shown in Figure 5 of Davis et al. (1995b) are redundant since the tip-tilt detection is now done on the red optical table (see Section 2.3) and they have been removed. These cubes had significant aberrations and their removal results in better performance of the blue system. The dispersing prisms have been mounted on rotating platforms driven by stepping motors to allow remote wavelength selection and rapid wavelength switching. The slits have also been fitted with stepping motors to permit remote control of the spectral bandwidth. The red and blue systems can now be operated simultaneously with the optical paths equalised for the red system by fringeenvelope tracking. This will not necessarily equalize the optical paths for the blue system and provision has been made for small adjustments to the relative paths of the north and south beams on the blue table. Referring to Figure 5 of Davis et al. (1995b), the mirror that directs the incoming north beam to the beam-combiner has been mounted on a slide driven by a stepping motor to enable small adjustments to the relative optical paths. The original reference detector on the blue table has been retained and enables the blue beams to be accurately superimposed with the aid of picomotor controlled mirror adjustments while the tip-tilt system stabilises the beams.

\section{Commissioning Results}

The corrections to the observational data for the residual effects of seeing, as applied in the data pipeline for the red system, have been discussed in Section 4.1 and an example of uncorrected and corrected data shown in Figure 9. As an example of the performance of the new red system 
Table 3. Observations of $\delta$ CMa

$b$ is the mean projected baseline which varied by a total of $\Delta b$ during the observations. $N$ is the number of sets of scans. Each set consisted of $1000140-\mu \mathrm{m}$ long scans, each scan consisting of 10240.2 -ms photon count samples. $V^{2}$ is the mean seeing-corrected normalised square of the fringe visibility. $\sigma_{V}^{2}$ is the scaled uncertainty in $V^{2}$ (details are given in the text)

\begin{tabular}{lrccccl}
\hline Date & \multicolumn{1}{c}{$\begin{array}{c}\Delta b \\
(\mathrm{~m})\end{array}$} & $\begin{array}{c}\Delta b \\
(\mathrm{~m})\end{array}$ & & $V^{2}$ & $\sigma_{V^{2}}$ & Calibrators \\
\hline 9 Nov 2003 & 5.00 & 0.006 & 4 & 0.980 & 0.020 & $\epsilon \mathrm{CMa}, \eta \mathrm{CMa}, \sigma^{2} \mathrm{CMa}$ \\
11 Nov 2003 & 19.96 & 0.004 & 2 & 0.582 & 0.026 & $\epsilon \mathrm{CMa}, \eta \mathrm{CMa} \sigma^{2} \mathrm{CMa}$ \\
12 Nov 2003 & 19.94 & 0.148 & 4 & 0.598 & 0.019 & $\epsilon \mathrm{CMa}, \eta \mathrm{CMa}, \sigma^{2} \mathrm{CMa}$ \\
27 Feb 2004 & 4.99 & 0.007 & 3 & 0.996 & 0.022 & $\epsilon \mathrm{CMa}, \eta \mathrm{CMa}, \sigma^{2} \mathrm{CMa}$ \\
28 Feb 2004 & 4.99 & 0.010 & 6 & 0.968 & 0.022 & $\epsilon \mathrm{CMa}, \eta \mathrm{CMa}$ \\
1 Mar 2004 & 39.94 & 0.060 & 3 & 0.048 & 0.006 & $\epsilon \mathrm{CMa}, \eta \mathrm{CMa}$ \\
30 Mar 2004 & 30.00 & 0.006 & 2 & 0.239 & 0.038 & $\epsilon \mathrm{CMa}, \eta \mathrm{CMa}$ \\
21 Apr 2004 & 4.99 & 0.012 & 4 & 0.961 & 0.017 & $\epsilon \mathrm{CMa}, \eta \mathrm{CMa}$ \\
9 Jan 2005 & 5.00 & 0.010 & 8 & 0.926 & 0.018 & $\epsilon \mathrm{CMa}, \eta \mathrm{CMa}$ \\
11 Dec 2006 & 29.96 & 0.066 & 8 & 0.230 & 0.004 & $\epsilon \mathrm{CMa}, \eta \mathrm{CMa}$ \\
12 Dec 2006 & 9.99 & 0.023 & 6 & 0.863 & 0.024 & $\epsilon \mathrm{CMa}, \eta \mathrm{CMa}$ \\
\hline
\end{tabular}

the determination of the angular diameter of $\delta \mathrm{CMa}$ is presented in Section 6.1. The results of tests carried out on the upgraded blue system are given in Section 6.2.

\subsection{The Angular Diameter of $\delta$ CMa}

The angular diameter of the bright southern supergiant $\delta$ CMa (F8 Iab) (HR 2693, HD 54065) has been measured with the Narrabri Stellar Intensity Interferometer (Hanbury Brown, Davis \& Allen 1974) and twice with SUSI at $442 \mathrm{~nm}$ (Davis et al. 1995b). For this reason it was chosen as an early observational target for the new red system to provide an illustration of its performance.

The observations of $\delta$ CMa were made using the $700 \mathrm{~nm}$ filters (see Section 3.3) employing 5, 10, 20, 30 and $40-\mathrm{m}$ baselines. Each observation of $\delta \mathrm{CMa}$ was bracketed by observations of calibrator stars. The primary calibrators were $\epsilon \mathrm{CMa}$ and $\eta \mathrm{CMa}$ and these were used on all twelve nights. $\sigma^{2} \mathrm{CMa}$ was also used as a calibrator on four nights of observation. The observational data were processed with the SUSI pipeline with seeing corrections (Section 4.1). Details of the observations and seeingcorrected calibrated values of $V^{2}$ are listed in Table 3 . Observations made in 2003 on four additional nights to those listed in Table 3 have been omitted. Unfortunately, for these early observations with the new system, on three of the nights insufficient seeing data were recorded for the data to be successfully processed. The fourth night involving observations at the $10-\mathrm{m}$ baseline, made under poor observing conditions, resulted in a value of $V^{2}$ lying some four sigma from a uniform-disk angular diameter fit to the other data and it has been omitted.

The uncertainties in the values of $V^{2}$ have been determined by scaling the standard errors in the mean (SEM), determined from the $N$ sets of scans for each night, by an appropriate value of student's $t$ to take into account the fact that they have been determined from a small number of independent measurements. The confidence interval chosen was $68 \%$, equivalent to that of the standard deviation for a Gaussian distribution. The scaled uncertainties $\left(\sigma_{V^{2}}\right)$ listed in Table 3 are the revised SEM values.

The angular diameter of the equivalent uniform disk $\theta_{\mathrm{UD}}$ was found by fitting the expression $\left|2 J_{1}(x) / x\right|^{2}$, where $x=\pi b \theta_{\mathrm{UD}} / \lambda_{\text {eff }}$, to the $V^{2}$ data. $b$ is the projected baseline and $\lambda_{\text {eff }}$ the effective wavelength of the observation. For $\delta \mathrm{CMa}$ the effective wavelength has been determined specifically for the star as it is a reddened supergiant. The flux distribution given by Kiehling (1987) for $\delta$ CMa has been used to calculate the effective wavelength, following the same procedure described in Section 3.3, to be $695.6 \pm 2.0 \mathrm{~nm}$. A direct measurement of the effective wavelength at a baseline of $5 \mathrm{~m}$ gave a value of $695.1 \mathrm{~nm}$. This differs from the computed value by only one quarter of the adopted uncertainty and, because of the large scatter shown in Figure 7 for measured values for main-sequence stars, the computed value has been adopted for the analysis.

The broad spectral bandwidth used for the observations will result in bandwidth smearing (Tango \& Davis 2002) arising from the variation in limb darkening as a function of wavelength across the band and from nonlinearity in the fitted curve. An investigation of this effect for $\delta \mathrm{CMa}$ showed that the effect was negligible for all baselines except $40 \mathrm{~m}$. At $40 \mathrm{~m}$ the combined effects of limb-darkening and curvature increased the observed $V^{2}$ by $1.7 \%$ relative to a monochromatic measurement at the effective wavelength. Therefore, the observed value of $V^{2}$ listed in Table 3 has been reduced by this amount prior to the fit for the uniform-disk angular diameter.

The uniform-disk angular diameter determined from the fit to the values of $V^{2}$ in Table 3 , with the value at $40 \mathrm{~m}$ reduced to 0.47 , is $3.457 \pm 0.019$ mas. It is noted that the effect of the change in value of $V^{2}$ for $40 \mathrm{~m}$ on the value for $\theta_{\mathrm{UD}}$ is only 0.001 mas. The fit also gives 


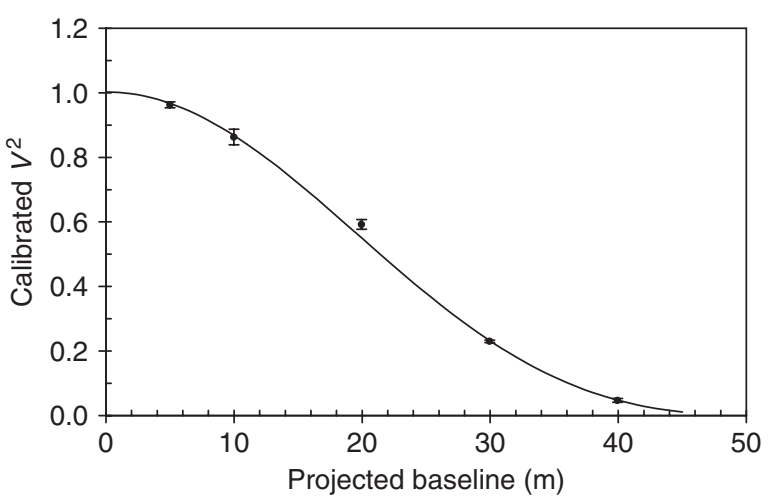

Figure 11 Weighted mean values of calibrated $V^{2}$ versus projected baseline for $\delta \mathrm{CMa}$. The curve is a least-squares fit to the observations for a uniform disk model (see text for details).

Table 4. The uniform-disk angular diameter of $\delta$ CMa determined with the NSII and with SUSI

\begin{tabular}{lccr}
\hline Instrument & $\begin{array}{c}\lambda \\
(\mathrm{nm})\end{array}$ & $\begin{array}{c}\theta_{\mathrm{UD}} \\
(\mathrm{mas})\end{array}$ & $\sigma \%$ \\
\hline NSII & 443.0 & $3.29 \pm 0.46$ & 14.0 \\
SUSI & 442.0 & $3.474 \pm 0.091$ & 2.6 \\
SUSI & 442.0 & $3.535 \pm 0.090$ & 2.5 \\
SUSI & 695.6 & $3.457 \pm 0.024$ & 0.8 \\
\hline
\end{tabular}

the $V^{2}$ value at zero baseline equal to $1.003 \pm 0.009$. The reduced $\chi^{2}$ for the fit is 1.73 indicating that the uncertainties in the measured values of $V^{2}$ are underestimated and this is believed to be due to incomplete correction of residual seeing effects. The uncertainties in the results of the fit have therefore been increased by $\sqrt{1.73}$. The uncertainty in the effective wavelength, given above, is $\sim \pm 0.3 \%$. Taking all uncertainties into account we find $\theta_{\mathrm{UD}}=3.457 \pm 0.024$ mas $( \pm 0.7 \%)$ and the zero baseline $V^{2}=1.003 \pm 0.011$

Weighted mean values for the $V^{2}$ at each baseline are plotted in Figure 11 together with the fitted curve.

The new value for the uniform-disk angular diameter is listed in Table 4 with the previous measurement made with the Narrabri Stellar Intensity Interferometer (Hanbury Brown, Davis \& Allen 1974) and the two measurements made at $442 \mathrm{~nm}$ with SUSI (Davis et al. 1999b). The uncertainty in the angular diameter has been steadily reduced. Note that, since the equivalent uniform-disk angular diameter is a function of wavelength, the values in Table 4 cannot be compared directly with one another.

The true, limb-darkened angular diameter of $\delta \mathrm{CMa}$, for each value of the uniform-disk angular diameters listed in Table 4, has been obtained using the appropriate correction factor interpolated from the tabulation of Davis, Tango \& Booth (2000). For this purpose the following physical parameters for $\delta$ CMa were adopted: effective temperature $T_{\text {eff }}=6000 \pm 200 \mathrm{~K}$ based on a number of values in the literature; $\log g=0.6$ and $[\mathrm{Fe} / \mathrm{H}]=0.19$ from Luck \& Lambert (1985). The effective temperature has
Table 5. The limb-darkened angular diameter of $\delta \mathrm{CMa}$

$\rho_{\lambda}$ is the ratio of limb-darkened to uniform-disk angular diameter from the tabulation by Davis, Tango \& Booth (2000) used to convert the uniform-disk angular diameters in Table 4 to the limb-darkened angular diameters in this table

\begin{tabular}{lccc}
\hline Instrument & $\begin{array}{c}\lambda \\
(\mathrm{nm})\end{array}$ & $\rho_{\lambda}$ & $\begin{array}{c}\theta_{\mathrm{LD}} \\
(\mathrm{mas})\end{array}$ \\
\hline NSII & 443.0 & 1.099 & $3.62 \pm 0.51$ \\
SUSI & 442.0 & 1.100 & $3.82 \pm 0.11$ \\
SUSI & 442.0 & 1.100 & $3.89 \pm 0.11$ \\
SUSI & 695.6 & 1.051 & $3.633 \pm 0.026$ \\
\hline
\end{tabular}

been determined subsequently with the aid of the new angular diameter to be $5818 \pm 53 \mathrm{~K}$ (Davis et al. 2007a). The limb-darkening correction factors were checked using the revised temperature with the same values for $\log g$ and $[\mathrm{Fe} / \mathrm{H}]$. The only change was for $695.6 \mathrm{~nm}$ with an increase from 1.050 to 1.051 . Although this has negligible effect, reducing the temperature by only $2 \mathrm{~K}$, the revised value has been used. The correction factors and the resulting values for the limb-darkened angular diameter are listed in Table 5. The accuracy of the correction factors depends on how well the models represent the star but we note that the interpolation in the tabulation by Davis, Tango \& Booth (2000) has an uncertainty of $\sim \pm 0.002$. The limb-darkened angular diameter values represent the same quantity and can be compared.

The uncertainty in the NSII value for the limb-darkened angular diameter is large and includes all three values determined with SUSI. However, the new $695.6 \mathrm{~nm}$ SUSI value differs from each of the $442 \mathrm{~nm}$ values by more than the sum of the individual uncertainties in each case. Although the two $442 \mathrm{~nm}$ values agree with one another we believe that the new value is the most reliable because of the improvement in calibration and seeing correction techniques that have been developed for the red system.

The blue measurements were made during the commissioning phase of SUSI and the analysis was based on observation and calibration procedures which have since been significantly improved as a result of experience. For example, the current observing procedure developed with the red system is to use at least two calibrators, preferably positioned either side of the programme star in the sky, to bracket all observations of the programme star between observations of calibrators, and to minimise the cycle time for a calibrator-programme star-calibrator sequence of observations to minimise the effects of changing seeing conditions. At the shorter blue wavelengths, where both spatial and temporal effects of seeing are greater, these changes in procedure are even more important. For the blue observations only one calibrator was used at a time and not all observations of $\delta \mathrm{CMa}$ were bracketed by the calibrator. The cycle time for a calibrator-programme star-calibrator sequence of 
observations averaged 57 minutes compared with 18 minutes for the new red measurement.

In view of these shortcomings the blue analysis was revisited and new fits to the data were made to determine the angular diameter. Observations which were not bracketed by calibrator observations were omitted and, in the case of observations calibrated by $\epsilon \mathrm{CMa}$, this reduced the number of individual values of $C$ from 127 to 94 and, in the case of $\eta \mathrm{CMa}$, from 94 to 88 . Fits were then made to the individual measurements of $V^{2}$, rather than to weighted means for each baseline as was done in the original analysis. The resulting values for the limbdarkened angular diameter of $\delta \mathrm{CMa}$ are, for $\epsilon \mathrm{CMa}$ as calibrator, $3.75 \pm 0.11$ mas and, for $\eta \mathrm{CMa}$ as calibrator, $3.70 \pm 0.17$ mas. While a discrepancy with the new value remains, it is $\sim 1.1 \sigma$ in one case and $\sim 0.4 \sigma$ in the other. The agreement between the original blue values in Davis et al. (1995b), which differed significantly from the new value, must be regarded as fortuitous and the reexamination of the blue data supports the conclusion that the new value is the most reliable because of the improvements in observation, calibration, and seeing correction techniques that have been developed for the red system.

A more detailed discussion of the results and the combination with flux distributions to determine the emergent flux and effective temperature for the star is published elsewhere (Davis et al. 2007a).

\subsection{Blue System Tests}

A number of tests have been carried out with the red and blue systems operating simultaneously to evaluate the performance of the updated blue system. These include the alignment of the beams in the blue system, adjusting the optical paths differentially in the blue system to ensure path equality at the beam-combiner, calibrating the wavelength scale, and correcting the blue data for differential optical path length variations and seeing effects using data from the red system. In each test the red system was continuously scanning the fringe envelope and providing feedback to the optical path length compensator to minimise the differential path length variations. The blue data were recorded and processed as discussed by Davis et al. (1995b).

\subsubsection{Beam Alignment for the Blue System}

The tip-tilt correction system aligns and maintains alignment of the beams for the red system but, for the blue system, the initial alignment of the beams must be carried out separately. Once aligned, the tip-tilt system will maintain the alignment for the blue system. The alignment is achieved using the original reference detector for the blue system (Davis et al. 1999a). The north and south beams are aligned one at a time by closing shutters to isolate the beam being aligned. The beam is then aligned on the reference detector, with the aid of either meters or an oscilloscope displaying the vertical and horizontal positions of the image, using the picomotor actuators on the back of the mirror directing the beam into the beam-combiner. Once

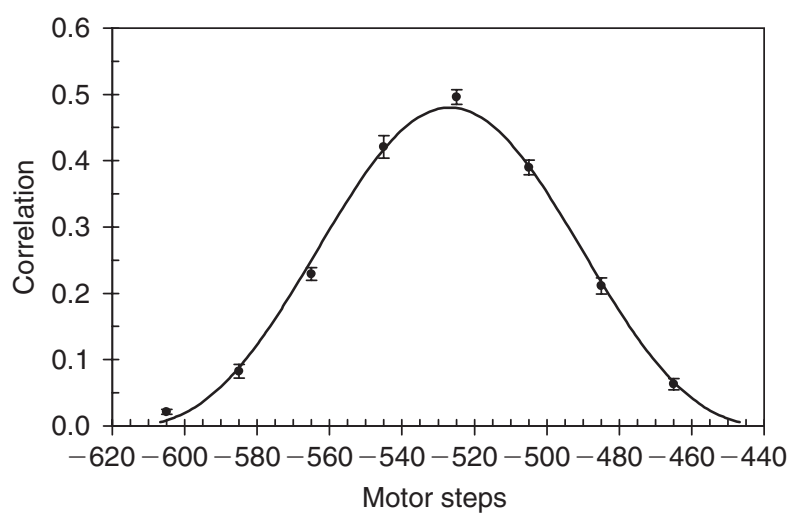

Figure 12 An example of a delay curve measured with the blue system for $\alpha$ Vir at a baseline of $5 \mathrm{~m}$. The curve is a fit to the raw measurements of $V^{2}$ and the position of the peak is $-526.8 \pm 0.6$ motor steps. Further details are given in the text.

aligned at the start of an observing night it is a matter of less than $\sim 30$ seconds to check the alignment during the course of the night. Experience has shown it to be extremely stable unless the differential optical path length is adjusted by moving the north input mirror on its slide (see Section 6.2.2).

\subsubsection{Optical Path Matching for the Blue System}

It was pointed out in Section 6.2 that matching the paths for the red system does not necessarily do the same for the blue system and there is likely to be a fixed optical path offset between the beams in the blue system. It was also noted that the mirror directing the north beam to the blue beam-combiner is mounted on a stepper motor driven slide. In order to establish the fixed optical path offset between the beams on the blue table, and hence the matched path condition for the blue system, the slidemounted mirror was stepped through the fringe envelope to produce a 'delay curve', the peak of which occurs at the matched path position. This was done with the red system maintaining matched optical paths on the red table. A typical delay curve is shown in Figure 12 in which the measured $V^{2}$ is plotted against the number of motor steps from the zero position of the slide. One motor step is equal to a differential change in optical path length of $1.73 \mu \mathrm{m}$. The measurements were made at a central wavelength of $480 \mathrm{~nm}$ with the slit width set to give a spectral bandwidth of $1.5 \mathrm{~nm}$. The fitted curve has the form of $|\sin x / x|^{2}$, corresponding to the near rectangular spectral bandpass defined by the slits in the blue system. We note that the translation of the slide does not maintain the orientation of the mirror precisely and small angular changes in the direction of the reflected beam occur. To overcome this the alignment of the north beam was checked, as described in Section 6.2.1, whenever the mirror was translated by $\geq 20$ steps. The realignment takes no more than 30 seconds and the fit of the delay curve in Figure 12 and the high value of $V^{2}$ for the peak of the curve are indicative of the success of this approach. 


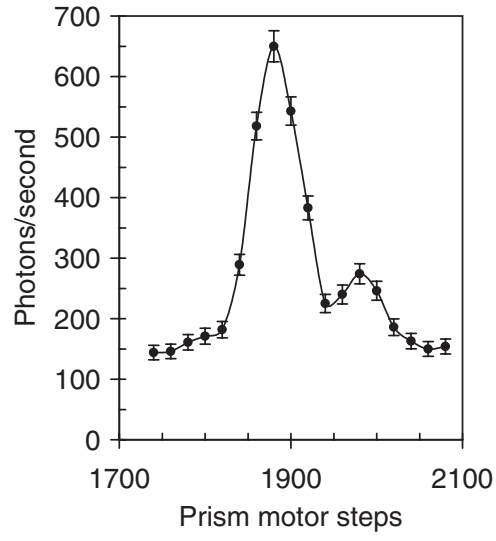

(a)

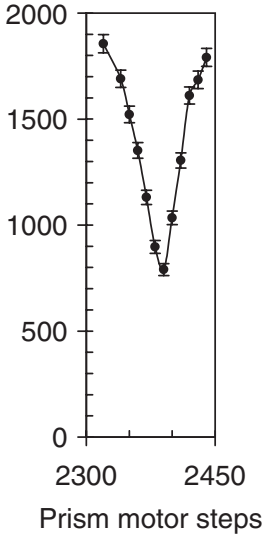

(b)
Figure 13 Scans through (a) the CIII-IV emission lines at $\lambda 465 \mathrm{~nm}$ for $\gamma^{2}$ Vel and (b) the core of the $\mathrm{H} \beta$ absorption line for $\alpha \mathrm{CMa}$ using the rotating prism mounts as described in the text. In each case the spectrograph slit was set to give a spectral bandpass $1 \mathrm{~nm}$ wide, corresponding to 20 prism motor steps.

\subsubsection{Calibration of the Wavelength Scale}

The wavelength is selected by rotating the prism mount and the general form of the relationship between wavelength and prism motor steps has been calculated. However, it needs to be calibrated with observations of sources of known wavelength. A combination of laser lines and stellar emission and absorption lines have been used and examples of stellar line observations are shown in Figure 13. In each case a $30 \mathrm{~s}$ integration was made at each position as the prism was rotated a few steps at a time.

\subsubsection{Correction of Blue-System Observations}

It was anticipated that optical path tracking errors and residual seeing errors would reduce the value of $V^{2}$ measured with the blue system. A number of observations have been made in which the integration time for the blue system matched the time of 200 seconds for a simultaneous set of scans with the red system. The loss in $V^{2}$ due to optical path tracking errors, which were derived from the recorded positions of the centres of the red-system fringe envelopes, was computed and a correction applied to the measured blue $V^{2}$. The seeing correction derived for the red observations was also applied as a correction to the blue $V^{2}$. The application of these two corrections significantly improved the stability of the blue measurements in all cases. An example is shown in Figure 14. The observations were made with a baseline of $20 \mathrm{~m}$ and a spectral bandwidth of $4 \mathrm{~nm}$ centred on a wavelength of $460 \mathrm{~nm}$. The seeing was good but variable and the improvement is clearly seen. The rms scatter of the data has been reduced from $\pm 9.8 \%$ to $\pm 2.9 \%$.

\subsection{Sensitivity Limits}

There are several factors that have contributed to an increase in sensitivity of the red system compared to the original blue system. These include the increased quantum

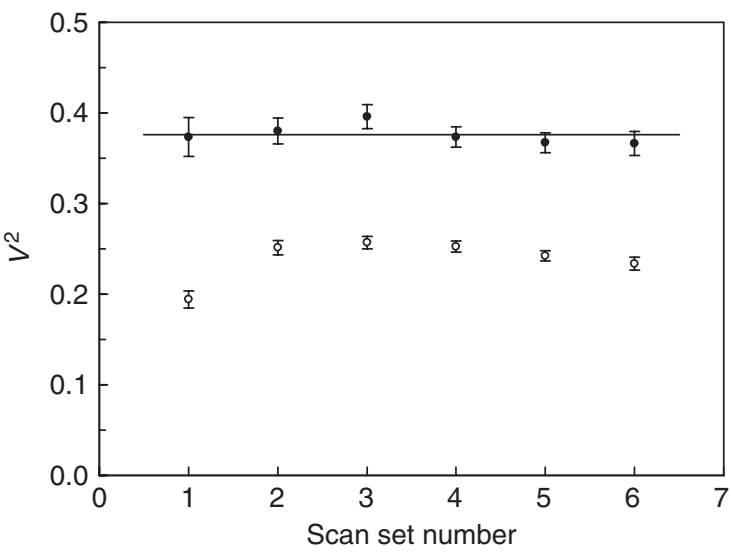

Figure 14 An example of the effect of applying corrections for differential optical path tracking errors and seeing effects. The data were obtained at a baseline of $20 \mathrm{~m}$ for $\beta \mathrm{Cru}$. The data points represented by open circles are the raw measurements of $V^{2}$. The filled circles represent the same data after correction. The horizontal line is the mean of the corrected data points. Further details are given in the text.

Table 6. A summary of the visual magnitude gain in sensitivity, $\Delta V$, of the red system compared to the original blue system as a function of spectral type

$V_{\text {limit }}$ (a) is the limiting visual magnitude for observations with the red system's $700 \mathrm{~nm}$ filter $\left(V_{\text {limit }}=+5\right.$ for spectral type A0) and $V_{\text {limit }}(\mathrm{b})$ is the limiting magnitude of the original blue system at $442 \mathrm{~nm}\left(V_{\text {limit }}=+2.5\right.$ for spectral type $\mathrm{A} 0)$

\begin{tabular}{llll}
\hline $\begin{array}{l}\text { Spectral } \\
\text { type }\end{array}$ & $\begin{array}{c}V_{\text {limit }} \\
\text { (a) }\end{array}$ & $\begin{array}{c}V_{\text {limit }} \\
\text { (b) }\end{array}$ & $\Delta V$ \\
\hline B0 & 4.7 & 2.8 & 1.9 \\
A0 & 5.0 & 2.5 & 2.5 \\
F0 & 5.3 & 2.2 & 3.1 \\
G0 & 5.6 & 1.9 & 3.7 \\
K0 & 5.8 & 1.7 & 4.1 \\
\hline
\end{tabular}

efficiency of the APD detectors compared to photomultipliers, the larger value of $r_{0}$ at the longer wavelength $\left(r_{0} \propto \lambda^{6 / 5}\right)$ allowing the use of larger apertures, and the use of wider spectral bandwidths. The limiting sensitivity for a point source at $700 \mathrm{~nm}$, which lies between the $R$ and $I$ photometric bands, is $\sim+5$. The gain in sensitivity relative to the blue system operating at $442 \mathrm{~nm}$ is a function of spectral type and is summarised in Table 6 .

The sensitivity of the upgraded blue system has yet to be established but a number of factors will contribute to its improvement. An increase in spectral bandwidth by up to a factor of 2.5 may be possible. The polarizing beam-splitters had aberrations due to inhomogeneities and no significant increase in signal-to-noise was obtained for effective aperture diameters on the sky greater than $\sim 40 \mathrm{~mm}$. Their removal allows larger aperture diameters and both polarizations to be used. It is estimated that a gain in sensitivity between 1.5 and 3 magnitudes will be obtained taking the limiting B magnitude to $>+4$. 


\section{Summary}

A beam-combination and detection system working at the red end of the spectrum $(\lambda \lambda 500-950 \mathrm{~nm})$ has been installed and commissioned in SUSI. It has provided an increase in sensitivity over the initial blue beamcombination and detection system that ranges from $\sim 2$ magnitudes at spectral type $\mathrm{B} 0$ to $>4$ magnitudes at $\mathrm{K} 0$. It has also resulted in improved calibration and accuracy of measurements of $V^{2}$ and hence improved accuracy in the determination of angular diameters. Dichroic beam-splitters have been introduced to allow simultaneous observations to be made with red and blue beamcombination systems. The blue beam-combination system has been upgraded to allow remote selection of wavelength in the range 430-500 $\mathrm{nm}$ and spectral bandpass in the range $1-10 \mathrm{~nm}$. Observations of $\delta \mathrm{CMa}$ made with the red system have been presented to illustrate the improvements achieved and the results of various tests on the two spectral systems have been discussed.

Results based on observations with the new red system include the angular pulsations of the Cepheids $\ell$ Car and $\beta$ Dor (Davis et al. 2007b), component mass and distance determinations for the spectroscopic binaries $\lambda$ Sco (Tango et al. 2006) and $\gamma^{2}$ Vel (North et al. 2007), and dust scattering in Miras resolved by optical interferometric polarimetry (Ireland et al. 2005). Both blue and red systems are now engaged in a programme of observations of single, binary, pulsating, and rapidly rotating stars.

\section{Acknowledgments}

The SUSI programme has been funded jointly by the Australian Research Council and the University of Sydney, with additional support from the Pollock Memorial Fund and the Science Foundation for Physics within the University of Sydney. M.J.I. acknowledges the support of an Australian Postgraduate Award, J.R.N. and A.P.J. the support of a University Postgraduate Award, and A.P.J. and S.M.O. the support of Denison Postgraduate Awards. J.C. was supported by MNRF and EBS by ARC funds.
Collaboration with the CHARA group of the Georgia State University and the exchange of ideas and the joint development of software, particularly through the contributions of T. A. ten Brummelaar, is acknowledged.

\section{References}

Baldwin, J. E., Boyson, R. C., Cox, G. C., Haniff, C. A., Rogers, J., Warner, P. J., Wilson, D. M. A. \& Mackay, C. D., 1994, in SPIE: Amplitude and Intensity Spatial Interferometry II, 2200, 118

Bracewell, R., 1965, The Fourier Transform and its Applications (New York: McGraw-Hill)

Davis, J., Ireland, M., Jacob, A. P., North, J., Owens, S. M., Robertson, J. G., Tango, W. J. \& Tuthill, P. G., 2006, in SPIE: Advances in Stellar Interferometry, Eds. Monnier, J. D., Schller, M. \& Danchi, W. C., 6268, 626804

Davis, J., Booth, A. J., Ireland, M. J., Jacob, A. P., North, J. R., Owens, S. M., Robertson, J. G., Tango, W. J. \& Tuthill, P. G., 2007a, PASA, 24, 151

Davis, J., Ireland, M. J., Jacob, A. P., North, J. R., Owens, S. M., Robertson, J. G., Tango, W. J. \& Tuthill, P. G., 2007b, in Proc. ESO Workshop 'The Power of Optical/IR Interferometry', Eds. Paresce, F. \& Richichi, A. (Berlin: Springer), in press

Davis, J., Tango, W. J. \& Booth, A. J., 2000, MNRAS, 318, 387

Davis, J., Tango, W. J., Booth, A. J., ten Brummelaar, T. A., Minard, R. A. \& Owens, S. M., 1999a, MNRAS, 303, 773

Davis, J., Tango, W. J., Booth, A. J., Thorvaldson, E. D. \& Giovannis, J., 1999b, MNRAS, 303, 783

Hanbury Brown, R., Davis, J. \& Allen, L. R., 1974, MNRAS, 167, 121

Ireland, M. J., 2005, PhD thesis, University of Sydney

Ireland, M. J., 2006, SPIE, 6268, 62680A-1

Ireland, M. J., Tuthill, P. G., Davis, J. \& Tango, W. J., 2005, MNRAS, 361,337

Kiehling, R., 1987, A\&AS, 69, 465

Luck, R. E. \& Lambert, D. L., 1985, ApJ, 298, 782

North, J. R., Tuthill, P. G., Tango, W. J. \& Davis, J., 2007, MNRAS, 377,415

Seneta, E. B., 1991, MSc thesis, University of Sydney

Silva, D. R. \& Cornell, M. E., 1992, ApJS, 81, 865

Tango, W. J. \& Davis, J., 2002, MNRAS, 333, 642

Tango, W. J. \& Twiss, R. Q., 1980, Progress in Optics, XVII, 239

Tango, W. J., et al., 2006, MNRAS, 370, 884

Tuthill, P. G., Davis, J., Ireland, M., North, J., O'Byrne, J., Robertson, J. G. \& Tango, W. J., 2004, in SPIE: New Frontiers in Stellar Interferometry, Ed. Traub, W. A., 5491, 499 